\title{
Risk Factors for Lymph Node Metastasis and Surgical Scope in Patients With Early-Stage Non- Small Cell Lung Cancer: A Single-Center Study in China
}

\section{Jianlong Bu}

Harbin Medical University Third Clinical College: Tumor Hospital of Harbin Medical University https://orcid.org/0000-0003-1379-8886

\section{Pinyi Zhang}

Harbin Medical University Third Clinical College: Tumor Hospital of Harbin Medical University

Xiaohong Wu

Harbin Medical University Third Clinical College: Tumor Hospital of Harbin Medical University

\section{Su Zhao}

Harbin Medical University Third Clinical College: Tumor Hospital of Harbin Medical University

\section{Sainan Pang}

Harbin Medical University Third Clinical College: Tumor Hospital of Harbin Medical University

Jinfeng Ning ( $\nabla$ ningjinfenghyd@163.com )

Harbin Medical University Third Clinical College: Tumor Hospital of Harbin Medical University

\section{Shidong Xu}

Harbin Medical University Third Clinical College: Tumor Hospital of Harbin Medical University

\section{Research Article}

Keywords: non-small cell lung cancer, NSCLC, lymph node metastasis, ground-glass opacity, GGO.

Posted Date: August 17th, 2021

DOI: https://doi.org/10.21203/rs.3.rs-730212/v1

License: (c) (i) This work is licensed under a Creative Commons Attribution 4.0 International License. Read Full License 


\section{Abstract \\ Background}

It is difficult to determine the lymph node metastasis of patients with early-stage non-small cell lung cancer (NSCLC) before surgery. The purpose of this study is to investigate risk factors of lymph node metastasis in early-stage NSCLC, thereby to identify the surgical indications for lymph node dissection in early-stage NSCLC.

\section{Methods}

We conducted a retrospective study of patients with tumor size $\leq 30 \mathrm{~mm}$ who underwent radical resection of NSCLC. Binary logistic regression analysis was applied to predict risk factors for lymph node metastasis, and subject operating characteristics (ROC) curve was used to evaluate the independent risk factors.

\section{Results}

Overall, 44 patients $(6.8 \%)$ with early-stage NSCLC had lymph node metastasis. Factors of tumor consolidation diameter $(p<0.001)$ and preoperative serum carcinoembryonic antigen (CEA) level $(p=$ 0.017) are independent risk factors lymph node metastasis in early-stage NSCLC. The ROC curve showed that the cut-off value of consolidation diameter was $16.5 \mathrm{~mm}$, and the area under the curve (AUC) was 0.825 ( $p<0.001,95 \%$ Cl: $0.780-0.870$ ); the cut-off value of serum CEA level was $1.765 \mu \mathrm{g} / \mathrm{L}, \mathrm{AUC}=0.661$ ( $\mathrm{p}<0.001,95 \% \mathrm{Cl}: 0.568-0.754)$. Moreover, 8 of 461 patients with tumor parenchyma $\leq 16.5 \mathrm{~mm}$ had lymph node metastasis, and 36 of 189 patients with tumor parenchyma $>16.5 \mathrm{~mm}$ had lymph node metastasis.

\section{Conclusion}

Tumor consolidation diameter and preoperative serum CEA are independent factors to predict early stage NSCLC. For patients with tumor parenchyma $>16.5 \mathrm{~mm}$, the probability of lymph node metastasis is higher and lobectomy plus lymph node dissection is recommended. For patients with tumor parenchyma $\leq 16.5 \mathrm{~mm}$, the probability of lymph node metastasis is lower and sublobectomy plus lymph node sampling is feasible.

\section{Introduction}

Lung cancer has become the most common malignant tumor in China with the highest morbidity and mortality, of which non-small cell lung cancer (NSCLC) accounts for $80 \%[1,2]$. The overall 5-year survival rate of lung cancer is $16 \%$, and the 5 -year survival rate of early-stage lung cancer patients after timely 
treatment can be increased to 50\% [3]. Recent developments in imaging modalities and the widespread application of low-dose helical computed tomography (CT) for lung cancer screening have led to an increase in the detection rate of early-stage lung cancer. Ground-glass opacity (GGO) is a slightly increased density on high-resolution computed tomography (HRCT) lung window, in which the bronchial and vascular textures are still visible [4]. Some patients with early-stage lung cancer appear as GGO on $\mathrm{CT}$, and patients with GGO-based early-stage lung adenocarcinoma have been reported to have a favorable prognosis after surgery [5]. The current standard method for early NSCLC surgery is lobectomy combined with complete lymph node dissection or sampling [6]. However, researches show that there are fewer cases of lymph node metastasis in GGO-based early-stage NSCLC [7, 8]. Therefore, whether patients with early-stage NSCLC need lymph node dissection or sampling has become a focus of attention.

In the past few decades, various biomarkeres have been explored for the prognosis of early stage NSCLC $[9,10]$. The predictive role of systemic inflammatory laboratory parameters, such as neutrophil to lymphocyte ratio (NLR), platelet to lymphocyte ratio (PLR), and lymphocyte to monocyte ratio (LMR), has also been confirmed in patients with NSCLC $[11,12]$. However, whether these inflammatory laboratory parameters have a predictive effect on the metastasis of NSCLC lymph nodes has been rarely reported.

This study aimed to investigate the factors that can predict early NSCLC lymph node metastasis, including basic characteristics of patients, imaging characteristics and preoperative examination of some hematological indicators. Therefore, we selected early-stage NSCLC patients with tumor diameter $\leq$ $30 \mathrm{~mm}$ and without imaging metastatic lymph nodes before surgery to study the incidence and risk factors for lymph node metastasis, thereby to provide the surgical indications for lymph node dissection in early-stage NSCLC.

\section{Methods}

\section{Patients}

A retrospective analysis was performed by using data collected from the clinical database of the department of thoracic surgery in Harbin Medical University Cancer Hospital. The study cohort was composed of consecutive patients scheduled to undergo initial surgery for NSCLC from January 2018 to December 2019. The inclusion criteria in this study was: (1) histopathologically confirmed primary NSCLC; (2) chest high-resolution CT (HRCT) was performed before operation; (3) the maximum diameter of tumor $\leq 30 \mathrm{~mm}$; (4) lobectomy and systematic lymph node dissection have been performed; (5) blood cell analysis and serum carcinoembryonic antigen (CEA) were performed before operation. Patients were excluded with the following conditions: (1) histopathologically confirmed carcinoma in situ; (2)history of neoadjuvant chemotherapy for NSCLC before operation; (3) history of malignant tumor; (4) preoperative chest CT showed mediastinal lymphadenopathy or hilar lymphadenopathy; (5) distant metastasis cannot be excluded. Ultimately, 650 patients were enrolled into the present study. This study was approved by the 
Institutional Ethics Review Board of Harbin Medical University Cancer Hospital. Informed written consent was obtained from all patients before the study.

\section{Preoperative examinations}

All the patients received pretreatment assessments including detailed clinical history, physical examination, a series of biochemical blood tests, and imaging examinations including magnetic resonance imaging (MRI) or CT of head, chest HRCT, upper abdominal ultrasound or $\mathrm{CT}$, emission computed tomography (ECT), supraclavicular lymph node ultrasound and cardiopulmonary function test. The NSCLC staging was based on the 8th edition of TNM Classification.

\section{Operations}

All patients in this study underwent single-port video-assisted thoracic surgery, and had no history of lung surgery or second surgery. If intraoperative frozen pathological results show that the lung tumor is malignant (non-small cell lung cancer), anatomical lobectomy and systematic lymph node dissection will be performed whether it is peripheral nodules or deep lesions. In our center, the surgical scope of systemic lymph node dissection includes 2-4 stations and 7-14 stations lymph nodes for right lung tumors, and 4-14 stations for left lung tumors. N2 is defined as 2-9 stations lymph nodes and N1 is defined as 1014 stations lymph nodes. The status of lymph node involvement was defined as pN0 (pathologically confirmed no lymph node metastasis), pN1 (pathologically confirmed any metastatic lymph node in 1014 stations) and pN2 (pathologically confirmed any metastatic lymph node in 2-9 stations).

\section{Pathology}

Tumor histological types were recorded by two experienced pathologists. Histopathological analyses were performed according to WHO criteria (3rd edition) [13]. Intraoperative pathological types are divided into adenocarcinoma, squamous cell carcinoma and other types of tumors (including large cell carcinoma, carcinoid, atypical carcinoid, poorly differentiated carcinoma and adenosquamous carcinoma, etc.). All resected lymph nodes, including mediastinal lymph nodes, hilar lymph nodes and intrapulmonary lymph nodes, need to be pathologically examinated after surgical dissection.

\section{Data collection}

The information we collected mainly included general information of patients, preoperative imaging examination, laboratory examination, and histopathological results. The general information of patients included age, gender and smoking history; preoperative imaging examination included maximum tumor diameter, maximum tumor consolidation diameter, tumor location and consolidation/tumor (C/T) ratio shown by chest HRCT; preoperative laboratory examination included NLR, PLR, LMR and serum CEA level. Histopathological results included intraoperative histopathological types, postoperative histopathological types and lymph node pathological results.

Patients were divided into two groups according to the age. One group was younger than 60 years old (age $\leq 60$ ), the other group was older than 60 years old (age $\$ 60)$. The tumor size was defined as the 
maximum dimension of the tumor on the lung windows. The consolidation size was defined as the maximum dimension of the solid component of the lung windows excluding GGO. The C/T ratio was defined as the maximum dimension of consolidation on the lung window setting divided by the maximum dimension of the tumor on the lung window setting. Pulmonary nodules were described as pure ggo $(\mathrm{C} / \mathrm{T}$ ratio $=0)$, mixed $\mathrm{GGO}(0<\mathrm{C} / \mathrm{T}$ ratio $<1)$ and solid nodules $(\mathrm{C} / \mathrm{T}$ ratio $=1)$.

\section{Statistics}

All data analysis applications are SPSS 18 software (SPSS, Inc., Chicago, IL, USA). Risk factors for lymph node metastasis were analyzed by Student's t-tests, Kolmogorov-Smirnov tests(K-S tests), $\chi 2$ tests, Fisher's exact test, and logistic regression. The cut-off value of serum CEA level and consolidation size is determined by the receiver operating characteristic (ROC) curve. The statistical significance is considered when $p<0.05$.

\section{Results}

\section{Patients information}

A total of 650 patients who had initial surgery for NSCLC were included in this study. There were 398 men and 252 women, with a mean age of 58.3 years (range, 30-82 years) at the first diagnosis. $186(28.6 \%)$ patients had a history of smoking. The general characteristics of the patients are listed in Table 1. 
Table 1

General characteristics and their relationship with lymph node metastasis (X2-test and Fisher's exact test)

\begin{tabular}{|c|c|c|c|c|c|}
\hline Variables & Total & pNO & $\mathrm{pN} 1+\mathrm{N} 2$ & $x^{2}$ & $\mathbf{p}$ \\
\hline All patients & 650 & $606(93.2 \%)$ & $44(6.8 \%)$ & & \\
\hline Gender & & & & 1.595 & 0.207 \\
\hline Female & 398 & $375(94.2 \%)$ & $23(5.8 \%)$ & & \\
\hline Male & 252 & $231(91.7 \%)$ & $21(8.3 \%)$ & & \\
\hline Age, years & & & & 0.794 & 0.435 \\
\hline$<60$ & 342 & $316(92.4 \%)$ & $26(7.6 \%)$ & & \\
\hline$\geq 60$ & 308 & $290(94.2 \%)$ & $18(5.8 \%)$ & & \\
\hline Smoking history & & & & 8.439 & 0.004 \\
\hline None & 464 & $441(95.0 \%)$ & $23(5.0 \%)$ & & \\
\hline Yes & 186 & $165(88.7 \%)$ & $21(11.3 \%)$ & & \\
\hline Pathological type & & & & 0.951 & $0.633^{*}$ \\
\hline Adenocarcinoma & 601 & $561(93.3 \%)$ & $40(6.7 \%)$ & & \\
\hline squamous cell carcinoma & 36 & $33(91.7 \%)$ & $3(8.3 \%)$ & & \\
\hline other types of malignant tumors* & 13 & $12(92.3 \%)$ & $1(7.7 \%)$ & & \\
\hline Tumor locations & & & & 6.247 & $0.178 *$ \\
\hline Right upper lobe & 243 & $232(95.5 \%)$ & $11(4.5 \%)$ & & \\
\hline Right middle lobe & 53 & $48(90.6 \%)$ & $5(9.4 \%)$ & & \\
\hline Right lower lobe & 99 & $92(92.9 \%)$ & $7(7.1 \%)$ & & \\
\hline Left upper lobe & 183 & 169(92.3\%) & $14(7.7 \%)$ & & \\
\hline Left lower lobe & 72 & $65(90.3 \%)$ & $7(9.7 \%)$ & & \\
\hline
\end{tabular}

\section{Frequency and distribution of lymph node metastasis}

A total of 11455 lymph nodes were removed from 650 patients, with an average of 17.6 lymph nodes removed per patient. There were 606 cases (93.2\%) without lymph node metastasis, 44 cases $(6.8 \%)$ with 
lymph node metastasis, 19 cases $(2.9 \%)$ with only N1 metastasis, 12 cases $(1.8 \%)$ with only N2 metastasis, and 13 cases (2.0\%) with both $\mathrm{N} 1$ and N2 metastasis, 17 cases $(2.62 \%)$ with the lymph node metastasis in the lung lobe (including station $12,13,14)$. There were 74 cases $(11.4 \%)$ of pure GGO patients, 239 cases $(36.8 \%$ ) of solid nodules, and 337 cases $(51.8 \%)$ of mixed GGO patients. There was no lymph node metastasis in patients with pure GGO, 9 cases $(9 / 337=3.8 \%)$ of mixed GGO patients had lymph node metastasis, and 35 cases $(35 / 239=14.6 \%)$ of solid nodule patients had lymph node metastasis.

\section{Risk factors of lymph node metastasis}

The chi-square test and Fisher's exact test was used to analyze the relationship between the patient's age, gender, smoking history, lesion location and intraoperative pathological type and lymph node metastasis. These results show that smoking history $(x 2=8.439, p=0.004)$ is related to lymph node metastasis (Table 1). $T$ test was used to analyze the relationship between the tumor size $(t=-6.24, p<0.001)$ and consolidation size $(t=-10.80, p<0.001)$ and lymph node metastasis, suggesting that the two factors are both related to lymph node metastasis (Table 2). K-S test was used to analyze the relationship between $\mathrm{C} / \mathrm{T}$ ratio, serum CEA level, NLR, PLR and LMR and lymph node metastasis. These results suggest that $C / T$ ratio $(z=3.082, p<0.001)$ and serum CEA level $(z=1.851, p=0.002)$ are related to lymph node metastasis (Table 3).

Table 2

General characteristics and their relationship with lymph node metastasis (Student's t-test)

\begin{tabular}{|lllll|}
\hline Variables & pN0 & pN1 + N2 & t & P \\
\hline Tumor size $(\mathrm{mm})$ & $17.13 \pm 6.41$ & $22.41 \pm 5.34$ & -6.24 & $<0.001$ \\
\hline Consolidation size $(\mathrm{mm})$ & $10.94 \pm 8.33$ & $20.82 \pm 5.64$ & -10.80 & $<0.001$ \\
\hline
\end{tabular}

pN0: Pathological diagnosis without lymph node metastasis. pN1 + N2: Pathological diagnosis with $\mathrm{N} 1$ and/or N2 metastasis. 
Table 3

General characteristics and their relationship with lymph node metastasis (K-S test)

\begin{tabular}{|lllll|}
\hline Variables & pN0 & pN1 + N2 & Z & P \\
\hline C/T ratio & $0.65(0.28,1.00)$ & $1.00(1.00,1.00)$ & 3.082 & $<0.001$ \\
\hline serum CEA level $(\mu \mathrm{g} / \mathrm{L})$ & $1.90(1.19,3.02)$ & $2.83(1.67,6.19)$ & 1.851 & 0.002 \\
\hline NLR & $1.63(1.27,2.19)$ & $1.79(1.20,2.29)$ & 3.082 & 0.748 \\
\hline PLR & $116.14(92.57,150.60)$ & $114.45(82.96,170.80)$ & 0.677 & 0.133 \\
\hline LMR & $5.11(3.86,7.90)$ & $5.03(3.43,6.21)$ & 0.984 & 0.287 \\
\hline $\begin{array}{l}\text { pN0: Pathological diagnosis without lymph node metastasis. pN1 + N2: Pathological diagnosis with } \\
\text { N1 and/or N2 metastasis. C/T ratio: consolidation size/tumor size ratio. CEA: carcinoembryonic } \\
\text { antigen. PLR: platelet-to-lymphocyte ratio. NLR: neutrophil-to-lymphocyte ratio. LMR: lymphocyte-to- } \\
\text { monocyte ratio. }\end{array}$ & & & \\
\hline
\end{tabular}

The relationship between smoking history, tumor size, consolidation size, $\mathrm{C} / \mathrm{T}$ ratio, serum CEA level, and lymph node metastasis was analyzed by binary logistic regression. The results show that consolidation size [odds ratio $(O R)=1.142, p<0.001$ ] and serum CEA level $(O R=1.091, p=0.017)$ are independent influencing factors of lymph node metastasis, and the possibility of lymph node metastasis increases with the consolidation size and the CEA value (Table 4). The ROC curve was used to analyze the consolidation size and serum CEA level (Fig. 1). The results showed that for the consolidation size of $16.5 \mathrm{~mm}$ is the best cut-off point, the area under the curve (AUC) was 0.825 ( $p<0.001,95 \% \mathrm{Cl}$ : $0.780-$ 0.870 ), with a sensitivity of $81.8 \%$ and a specificity of $74.8 \%$; serum CEA level of $1.765 \mu \mathrm{g} / \mathrm{L}$ is the best cut-off point, AUC $=0.661(\mathrm{p}<0.001,95 \% \mathrm{Cl}: 0.568-0.754)$, with a sensitivity of $75 \%$ and a specificity of $46.4 \%$.

Table 4

Independent risk factors of lymph node involvement by multivariate analysis

\begin{tabular}{|llll|}
\hline Variables & Odds ratio & $95 \% \mathrm{Cl}$ & $\mathbf{p}$ \\
\hline Consolidation size & 1.142 & $1.094-1.193$ & $<0.001$ \\
\hline serum CEA level & 1.091 & $1.015-1.172$ & 0.017 \\
\hline \multicolumn{2}{|l}{ Cl: confidence interval. } & & \\
\hline
\end{tabular}

There are 189 patients with tumor consolidation size $>16.5 \mathrm{~mm}$. Among them, 36 patients $(36 / 189=$ $19.0 \%$ ) had lymph node metastasis; there were 461 patients with tumor consolidation size $\leq 16.5 \mathrm{~mm}$, including 8 cases had lymph node metastasis $(8 / 461=1.7 \%)$. 


\section{Discussion}

Although lobectomy plus lymph node dissection or sampling is still the standard surgical method for NSCLC [6], studies have shown that stage IA NSCLC and GGO-based lung adenocarcinoma have no diffenrences in survival time between the lobectomy group and the sublobectomy group $[8,14]$. These findings indicate that the tumor of patients with early-stage NSCLC can be completely removed by sublobectomy, which can achive a similar prognosis to the procedure of lobectomy. In fact, sublobectomy inevitably leads to some of the lymph nodes in the lung lobes that cannot be removed. To some extent, it can be concluded that the probability of N1 metastasis is extremly low in early-stage NSCLC [15].

Early-stage NSCLC usually does not find mediastinal lymph node enlargement (metastasis) in preoperative imaging examination, but a few patients appear to have mediastinal lymph node metastasis in postoperative pathology reports[16]. Similarly, Our study found that 25 patients (3.8\%) who did not prompt mediastinal lymph node metastasis before surgery had N2 metastasis. In this study, 12 (1.8\%) patients had single $\mathrm{N} 2$ metastasis, of which 3 patients had the smallest consolidation size of $10 \mathrm{~mm}$, and the tumors were all located in the upper lobe of the left lung (Patient One had 1 lymph node metastasis in 6 stations, and Patient Two had 1 lymph node metastasis in 6 stations and 1 lymph node in 9 stations, Patient Three had 1 lymph node metastasis in 5 stations and 1 lymph node metastasis in 6 stations). In consistent with the study by Wang et al. [17] reporting $1.5 \%$ of single N2 metastasis, our fingdings suggest that N2 metastasis may appear in patients with early-stage NSCLC, and the absence of N1 metastasis cannot completely exclude N2 metastasis. Alternatively, it can be reckoned that patients with early NSCLC may have N2 metastasis without N1 metastasis.

Mediastinal lymph node dissection can remove mediastinal lymph nodes and clarify the stage of lung cancer, providing a basis for the formulation of postoperative treatment plans for patients, but the mediastinal lymph node dissection would not only prolong the operation time, but also bring additional surgical risks [18]. Studies have confirmed that there are fewer cases of lymph node metastasis in pure GGO or GGO-based lung cancer [8]. Thus, if the method of screening patients with potential lymph node metastasis without increasing trauma can be applied before surgery, the surgical trauma to patients without lymph node metastasis will be reduced, and it will play a guiding role in the selection of surgical methods for physicians.

This study retrospectively analyzed the basic characteristics of early-stage NSCLC patients (age, gender, smoking history), imaging informations (focus location, tumor size, consolidation size, $\mathrm{C} / \mathrm{T}$ ratio), and preoperative blood tests (serum CEA level, NLR, PLR, LMR) and the relationship between intraoperative pathological types and lymph node metastasis. We found that the consolidation size and preoperative serum CEA level are independent Risk factors of lymph node metastasis. Our results suggest that the frequency of lymph node metastasis increases as the consolidation size of tumor increases. This result is consistent with the results of Murakawas et al. [19]. For consolidation size to predict lymph node metastasis, we obtained AUC $=0.825$ through ROC curve, the best cut-off point is $16.5 \mathrm{~mm}$, the sensitivity is $81.8 \%$, and the specificity is $74.8 \%$. Based on the best cut-off point, we found that for patients with 
consolidation size $>16.5 \mathrm{~mm}$, the probability of lymph node metastasis is $19.0 \%(36 / 189)$, and for patients with consolidation size $\leq 16.5 \mathrm{~mm}$, the probability of lymph node metastasis is $1.7 \%(8 / 461)$.

Thus, the factor of consolidation size is more accurate to predict lymph node metastasis. To some extent, it also confirms that the T-stage measurement of consolidation size in the 8th edition of TNM staging may be more accurate for tumor staging. Using ROC curve analysis for serum CEA level, AUC $=0.661$, the accuracy rate is not very ideal. In the ROC curve, we found that when the consolidation size is $9.5 \mathrm{~mm}$, it is the maximum predictive value with $100 \%$ sensitivity, and the specificity at this point is $48.3 \%$. The patients included in this experiment were not found lymph node metastasis when the consolidation size was less than $9.5 \mathrm{~mm}$, while just one study found $\mathrm{N} 1$ metastasis in $5 \mathrm{~mm}$ solid nodule [20]. Thus, it is difficult to find an absolute boundary of consolidation size to predict lymph node metastasis in NSCLC. In this research, no lymph node metastasis was found in those with pure GGO, which is consistent with the results of study by Zha JY et al.[21]. Until now, systematic lymph node dissection emphasizes the dissection of mediastinal lymph nodes (N2) and hilar lymph nodes. Currently, the clinical postoperative pathological results may not show all the metastastic lymph nodes in lung lobes. Thus, we did not discuss the relevant factors of intrapulmonary lymphatic metastasis. However, a complete N1 (especially the lymph nodes in the lung lobe) dissection would play a critical role in sublobectomy for early-stage lung cancer.

Immune cells, such as neutrophils and lymphocytes, with tumor cells and stromal cells constitute the microenvironment of tumor, and play an important role in the process of tumor development and progression [22, 23]. In addition, an experimental study showed that all tumor associated immune cells (except MUM1 + cells) in stage $₫$ tumor specimens were significantly higher than those in stage I specimens [24]. We tried to detect whether NLR, PLR, LMR and other indicators could predict lymph node metastasis. Although there was no obvious correlation in the statistical results, it cannot be ruled out that other immune cell or blood test indicators could predict lung cancer lymph node metastasis.

Despite the previous findings, this study still possesses some limitations. First, our clinical database of early-stage NSCLC, as a single-center administrative database, cannot capture every subtle factor, some of which may be critical for clinicians. Thus, further multi-center randomized trials are needed to verify these results. Second, we had little information on disease-free survival because of the lack of recurrence records based on long-term follow-up, which failed to evaluate the prognostic significance of lymph node dissection. Further investigation for us will also involve clinical research on the pathways and mechanisms of NSCLC lymph node metastasis before strict indications for prophylactic lymph node dissection for early-stage NSCLC are formally defined.

\section{Conclusion}

In conclusion, the present study demonstrated that consolidation size and serum CEA level are independent predictors of lymph node metastasis of non-small cell lung cancer with tumor size $\leq 30 \mathrm{~mm}$. while the tumor consolidation diameter has a high accuracy in predicting lymph node metastasis, and the preoperative serum CEA level has a low accuracy in predicting lymph node metastasis. Patients with 
consolidation size $>16.5 \mathrm{~mm}$ are found to have higher probability of lymph node metastasis and are recommended to receive lobectomy plus mediastinal lymph node dissection. For patients with NSCLC with consolidation size $\leq 16.5 \mathrm{~mm}$, lower probability of lymph node metastasis is found and sublobectomy together with mediastinal lymph node sampling can be performed.

\section{List Of Abbreviations}

area under the curve, AUC

carcinoembryonic antigen, CEA

emission computed tomography, ECT

ground-glass opacity, GGO

high-resolution computed tomography, HRCT

lymphocyte to monocyte ratio, LMR

magnetic resonance imaging, MRI

neutrophil to lymphocyte ratio, NLR

non-small cell lung cancer, NSCLC

platelet to lymphocyte ratio, PLR

subject operating characteristics, ROC

\section{Declarations}

\section{Ethics approval}

This study was approved by the Institutional Ethics Review Board of Harbin Medical University Cancer Hospital. Informed written consent was obtained from all patients before the study.

\section{Consent for publication}

None

\section{Availability of data and materials}

None

\section{Competing interests}


The authors declared no potential conflicts of interest with respect to the research, authorship, and/or publication of this article.

\section{Funding}

The present study was supported by "Chunhui plan" project of the Ministry of education of China, No. HLJ2019011 and Individualized and precise treatment of lung cancer, No. Nn10py2017-04.

\section{Author's contributions}

(I) Conception and design: Bu jianlong, Ning Jinfeng; (II) Administrative support: Ning Jinfeng, Xu Shidong; (III) Provision of study materials or patients: Bu Jianlong, Zhao Su, Ning Jinfeng, Xu Shidong; (IV) Collection and assembly of data: Bu Jianlong, Zhang Pinyi, Wu Xiaohong, Zhao Su; (V) Data analysis and interpretation: Bu Jianlong, Zhang Pinyi; (VI) Manuscript writing: All authors; (VII) Final approval of manuscript: All authors.

\section{Acknowledgements}

Not applicable

\section{References}

1. Cancer incidence. and mortality in China, 2015[J].JNCC, 2020.

2. Siegel R, Naishadham D, Jemal A. Cancer statistics, 2012. CA Cancer J Clin. 2012;62:10-29.

3. Chheang S, Brown K. Lung Cancer staging: clinical and radiologic perspectives [J]. Semin Interv Radiol. 2013;30(2):99-113.

4. Hansell DM, Bankier AA, MacMahon $\mathrm{H}$, et al. Fleischner Society: glossary of terms for thoracic imaging. Radiology. 2008;246:697-722.

5. Wilshire CL, Louie BE, Manning KA, et al. Radiologic Evaluation of Small Lepidic Adenocarcinomas to Guide Decision Making in Surgical Resection. Ann Thorac Surg. 2015;100:979-88.

6. Network NCC Non-small Cell Lung Cancer (Version 2. 2019). Available online: https://www.nccn.org/ professionals/physician_gls/pdf/nscl.pdf (accessed on 21 November 2019).

7. Suzuki K, Asamura H, Kusumoto M, et al. "Early" peripheral lung cancer: prognostic significance of ground glass opacity on thin-section computed tomographic scan. Ann Thorac Surg. 2002;74:16359.

8. Tsutani $\mathrm{Y}$, Miyata $\mathrm{Y}$, Nakayama $\mathrm{H}$, et al. Appropriate sublobar resection choice for ground glass opacity- dominant clinical stage IA lung adenocarcinoma: wedge resection or segmentectomy. Chest. 2014 Jan;145(1):66-71.

9. O’Byrne KJ, Ga emeier U, Bondarenko I, Barrios C, Eschbach C, Martens UM, et al. Molecular biomarkers in non-small-cell lung cancer: a retrospective analysis of data from the phase 3 FLEX study. Lancet Oncol. 2011;12:795-805. 
10. Douillard JY, Shepherd FA, Hirsh V, Mok T, Socinski MA, Gervais R, et al. Molecular predictors of outcome with gefitinib and docetaxel in previously treated non-small-cell lung cancer: data from the randomized phase âđç INTEREST trial. J Clin Oncol. 2010;28:744-52.

11. Cannon NA, Meyer J, lyengar P, Ahn C, Westover KD, Choy H, et al. Neutrophil-lymphocyte and platelet-lymphocyte ratios as prognostic factors after stereotactic radiation therapy for early-stage non-small-cell lung cancer. J Thorac Oncol. 2015;10:280-5.

12. Song $Y$, Wang $L$, Hong $Y$, et al. Lymphocyte to monocyte ratio is associated with response to first-line platinum-based chemotherapy and prognosis of early-stage non-small cell lung cancer patients. Tumour Biol. 2015;37:5285-93.

13. Travis WD, Organization WH, International Agency for Research on Cancer, International Association for the Study of Lung Cancer. International Academy of Pathology, Pathology and Genetics of Tumours of the Lung, Pleura, Thymus and Heart. Oxford University Press, Lyon, Oxford: IARC Press; 2004. (distributor).

14. Altorki NK, Yip R, Hanaoka T, et al. Sublobar resection is equivalent to lobectomy for clinical stage 1 a lung cancer in solid nodules. J Thorac Cardiovasc Surg. 2014 Feb;147(2):754-62.

15. Ye B, Cheng $M, G e X$, et al. Factors that predict lymph node status in clinical stage T1aNOMO lung adenocarcinomas. World J Surg Oncol. 2014 Feb;21:12: 42.

16. Suzuki K, Nagai K, Yoshida J, et al. The prognosis of surgically resected N2 non-small cell lung cancer: the importance of clinical N status. J Thorac Cardiovasc Surg. 1999;118:145-53.

17. Wang Y, Jing L, Wang G. Risk factors for lymph node metastasis and surgical methods in patients with early- stage peripheral lung adenocarcinoma presenting as ground glass opacity. $\mathrm{J}$ Cardiothorac Surg. 2020 Aug 12;15(1):121.

18. Moon $Y$, Sung SW, Namkoong M, et al. The effectiveness of mediastinal lymph node evaluation in a patient with ground glass opacity tumor. J Thorac Dis, 2016 sep, 8(9): 2617-2625.

19. Murakawa $T$, Konoeda $C$, Ito $T$, et al. The ground glass opacity component can be eliminated from the T-factor assessment of lung adenocarcinoma. Eur J Cardiothorac Surg. 2013 May;43(5):925-32.

20. Sakairi Y, Wada H, Fujiwara T, et al. The probability of nodal metastasis in novel T-factor: the applicability of sublobar resection. J Thorac Dis. 2019 Oct; 11(10): 4197-4204.

21. Zha J, Xie D, Xie H, et al. Recognition of "aggressive" behavior in "indolent" ground glass opacity and mixed density lesions. J Thorac Dis. 2016 Jul; 8(7):1460-1468.

22. Bollrath J, Greten FR. IKK/NF-kappaB and STAT3 pathways: Central signalling hubs in inflammationmediated tumour promotion and metastases. EMBO Rep. 2009;10:1314-9.

23. Ruffini E, Asioli S, Filosso PL, Lyberis P, Bruna MC, Macrì L, et al. Clinical significance of tumorinfiltrating lymphocytes in lung neoplasms. Ann Thorac Surg. 2009;87:365-72.

24. Banat GA, Tretyn A, Pullamsetti SS, Wilhelm J, Weigert A, Olesch C, et al. Immune and inflammatory cell composition of human lung cancer stroma. PLoS One. 2015;10:0139073. 
Figures
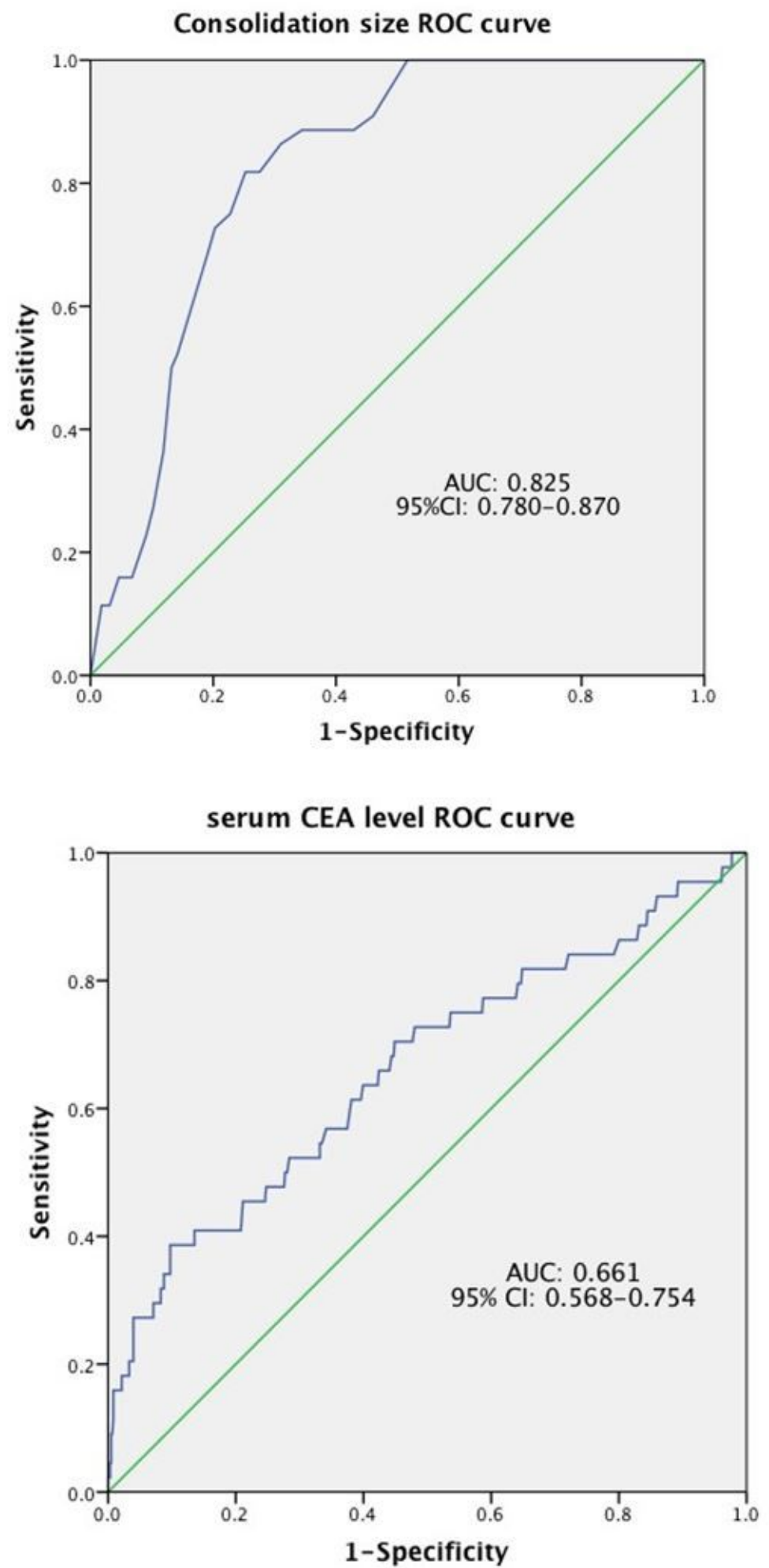

\section{Figure 1}

Receiver operating characteristic (ROC) curve of consolidation size and serum CEA level values in predict lymph node metastasis. The AUC for consolidation size was 0.825 ( $p<0.001,95 \% \mathrm{Cl}$ : $0.780-0.870)$ with a 
sensitivity of $81.8 \%$ and specificity of $74.8 \%$. The AUC for scrum CEA level was $0.661(p<0.001,95 \% \mathrm{Cl}$ : $0.568-0.754$ ) with a sensitivity of $75 \%$ and specificity of $46.4 \%$. 